\title{
Correlating Electronic Structure and Chemical Durability of Sulfonated Poly(Arylene Ether Sulfone)s
}

\author{
Jimmy Lawrence ${ }^{1,2}$, Koichi Yamashita ${ }^{1}$, Takeo Yamaguchi ${ }^{1,3 *}$ \\ ${ }^{1}$ Department of Chemical System Engineering, The University of Tokyo \\ 7-3-1 Hongo, Bunkyo-ku, Tokyo, Japan 113-8656
}

${ }^{2}$ Department of Polymer Science and Engineering, University of Massachusetts, 120

Governors Drive, Amherst, MA 01375, USA

${ }^{3}$ Tokyo Institute of Technology

Chemical Resources Laboratory

R1-17, 4259 Nagatsuta, Midori-ku, Yokohama 226-8503, JAPAN

Tel: $+81-45-924-5254$

Fax: +81-45-924-5253

* E-mail: yamag@res.titech.ac.jp 


\begin{abstract}
Many different proton-conducting polymeric materials have been developed for polymer electrolyte membrane fuel cells (PEMFCs). The development of perfluorosulfonic acidbased, polymer electrolyte membranes (PFSA-PEMs) was followed by aromatic hydrocarbon-based PEMs (HC-PEMs), which allow for tailored design and optimization of their molecular structures. Although many new PFSA-PEMs and HC-PEMs have shown promising proton conductivity and thermal stability, chemical degradation of these materials in an oxidizing environment remains a significant technical barrier in PEMFC development. Here, we used accelerated degradation tests and electronic structure analysis to examine the chemical stability of sulfonated poly(arylene ether sulfone) (SPES) copolymers, a highly thermally stable HC-PEM. HOMO levels, the presence of main chain-protecting steric groups, and HOMO-LUMO location along the main chain have significant effects on the chain scission modes and degradation rate of SPES copolymers. Rational design of HC-PEMs to suppress midpoint scission can open many opportunities in the development of highly robust polymer electrolytes for fuel cell and other energy storage applications.
\end{abstract}

\title{
Keywords
}

Polymer electrolyte membrane; accelerated test; electronic structure; molecular orbital; degradation mechanism; fuel cell 


\section{INTRODUCTION}

Over the past two decades, significant advances have been made in the area of effective material utilization (e.g., catalysts) and cell performance (e.g., proton conductivity) to make polymer electrolyte membrane fuel cells (PEMFCs) economical and useful for a broad range of applications.[1] Extensive collaborative projects were established to commercialize PEMFCs by 2012; however, the project goals of achieving $\$ 5-7 / \mathrm{kW}$ and cell lifetime of $5000 \mathrm{~h}$ have not been met.[2] The US Department of Energy report[2] cited the inadequate durability of fuel cell systems and inadequate understanding of the component degradation and failure mechanisms. Indeed, chemical stability is the most significant technical challenge for PEMFCs.[3]

The chemical stability of a PEMFC is determined by its most critical component, the membrane-electrode assembly (MEA). Within the MEA unit, many electrochemical reactions take place to convert the fuels introduced at the electrode inlets to electricity. Such reduction-oxidation reactions were also shown to produce reactive oxygen species (ROS) (e.g., $\mathrm{H}_{2} \mathrm{O}_{2}, \bullet \mathrm{OH}, \mathrm{HOO} \bullet$ ), which are formed as redox reaction by-products from two-electron reactions.[4] Although studies suggested different origin sites for the ROS, i.e., anode,[5] cathode,[6,7] or both anode and cathode,[8] these studies pointed to ROS attacks generated from $\mathrm{H}_{2} \mathrm{O}_{2}$ to be the cause of polymer electrolyte membrane (PEM) degradation. Such chemical degradation is exacerbated in the presence of metal ion contaminants from corroded metal bipolar plates (Fenton reaction),[9] and at higher operating temperature (to enhance the reaction kinetics and suppress catalyst poisoning).[3] Increasing the operating temperature of a PEMFC to medium-high temperatures $\left(>100{ }^{\circ} \mathrm{C}\right)$ is desirable to enhance electrocatalysis, mass-transport 
properties, and to eliminate problems from fuel impurities.[10,11] However, the thermal stability of the standard perfluorosulfonic acid-based, first-generation PEM (PFSA-PEM) is insufficient $\left(<90{ }^{\circ} \mathrm{C}\right)$ for such a purpose,[12] and therefore, the development of a thermally stable and chemically robust PEM suitable for medium-high temperature PEMFCs is highly important.

Highly thermally stable aromatic hydrocarbon-based PEMs (HC-PEMs) having remarkable proton conductivity $\left(\sigma_{\mathrm{HC}-\mathrm{PEM}}: \approx 0.4 \quad \mathrm{~S} / \mathrm{cm}, \quad \sigma_{\mathrm{Nafion} \circledast}: \quad 0.1 \quad \mathrm{~S} / \mathrm{cm}\right)$, thermomechanical stability (up to $140{ }^{\circ} \mathrm{C}$ ), and mass-transport properties have been developed through postsulfonation of thermoplastics, and copolymerization of sulfonated and nonsulfonated monomer/oligomers.[13-18] HC-PEMs have become promising replacement materials for PFSA-PEMs because of available and emerging synthetic approaches to design the polymer structures and properties.[14,19] However, the chemical stability of most HC-PEMs is still poor (fuel cell lifetime: 500-5000 h) and often not studied.[12] Several mitigation strategies to alleviate chemical degradation because of ROS attacks have been proposed,[9] such as blending PEM with free radical stabilizers,[16] sacrificial polystyrene layer,[20] crosslinkers,[21] and ceria nanoparticles.[22] Although the latter strategy suppressed the extent of PEM degradation and material leaching, the long-term transport properties of such ceria-containing MEAs are unknown.[23]

We previously showed that the dominant degradation pathway (i.e., unzip scission, midpoint scission) of sulfonated poly(arylene ether sulfone) (SPES) copolymers is influenced by the structures of their dihydroxy comonomers.[24] Such influence from the dihydroxy comonomer structure was also observed in the performance of SPES-based 4 
PEMs. SPES having 4,4'-biphenol, bisphenol A, bisphenol 6F and hydroquinone as the dihydroxy comonomers exhibited different water uptake, membrane stability and ion exchange capacity.[25] Interestingly, SPES having bisphenol $6 \mathrm{~F}$ as the dihydroxy comonomer showed better adhesion and electrochemical compatibility with Nafion based electrodes. Synthetic strategies to suppress the oxidative degradation of SPES-based HCPEMs described changing the position of electron withdrawing functional groups on the aromatic rings to tune the electron density of the polymer backbone,[26] and introducing aliphatic or other functional groups to lower the hydrophilicity of the polymer backbone.[27-29] The lifetime of PEMFCs prepared from SPES having fluorenyl dihydroxy comonomers reached $5,000 \mathrm{~h}$ under $90 \%$ relative humidity although it decreased to $2350 \mathrm{~h}$ under $60 \%$ relative humidity condition.[30] Therefore, methods to screen out promising comonomers and estimate the durability of SPES from their molecular structures are beneficial for the purpose of suppressing the dominant degradation pathway of SPES.

Fully sulfonated PESs were chosen as model compounds in this study because the hydrophilic component of PEM is more exposed to the solvent and thus more susceptible to the highly reactive ROS formed in the MEA.[24] Making fully water-soluble samples also eliminates other experimental variables, such as diffusivity (e.g., membrane thinning), membrane morphology (e.g., pinhole formation),[30] and the state of water in polymers.[31] We first examined the degradation of four types of SPES copolymers, correlated their chemical stability to their respective electronic structures, and then investigated the chemical stability of another two types of SPES copolymers to confirm our hypothesis. 
Electronic structure calculations beyond small molecules have been advanced by many breakthroughs in computational chemistry methods and parallel computing. Semiempirical theory,[32] density functional theory (DFT) and multilayered ab initio calculations (QM/MM) have been rigorously developed to analyze the molecular orbitals (MOs) from small molecules to biomolecules (enzymes).[33] Pioneered by Fukui et al., information from frontier MOs (highest occupied MO (HOMO) and lowest unoccupied MO (LUMO)) can be used to estimate the electrophilic, nucleophilic, and radical susceptibility of a given molecule.[34] Here, we used frontier MO level calculations and analysis to examine the attacks of $\bullet \mathrm{OH}$, the most reactive ROS,[35] on SPES copolymers. Frontier MO calculations have proved useful in many studies of ROS attacks. Cheng et al. performed a quantitative structure-property relationship analysis to correlate the HOMO level of phenol derivatives and their reaction rate with $\bullet$ OH.[36] Similarly, Parra et al. reported a HOMO level-based empirical model to estimate the photodegradation rate of herbicides having different substituents.[37]

We employed semiempirical theory (PM3) in the calculations because it allows for geometry optimization and electronic structure calculations of SPES monomers and oligomers to be computed and compared, within resources and a reasonable time frame. Calculation of the stabilization energy of $\mathrm{OH}$ radical adducts and intrinsic reaction coordinate (IRC) trajectories were performed on a SPES monomer (one repeating unit). MO calculations were also performed on SPES dimers and tetramers (two and four repeating units) to decrease the extent of terminal atom effects. We show later that such calculations on larger structures allowed for insights into the dominant chain scission modes of the polymers, and better agreement between experimental and theoretical 
results. To our knowledge, this is the first report correlating the electronic structure of sulfonated oligomers to their oxidative stability.

\section{EXPERIMENTAL SECTION}

The sodium salt of 3,3'-disulfonic acid-4,4'-dichlorodiphenyl sulfone (DSDCDPS) were supplied by Hitachi Chemicals (Japan). Bisphenol-A (BPA, $\geq 99 \%$ ), 4,4'thiodiphenol (TDP, 99\%) and 4,4'-(hexafluoroisopropylidene)diphenol (BP6F, 97\%) were purchased from Sigma-Aldrich (Japan). 4,4'-biphenol (BP, >99\%), 4,4'dihydroxydiphenylsulfone (BPS, 99\%) were purchased from TCI Chemicals (Japan). Hydrochloric acid ( $\mathrm{HCl}, 6 \mathrm{M}), 4,4^{\prime}$-dihydroxy benzophenone (BPn), isopropyl alcohol (IPA), hydrogen peroxide $\left(\mathrm{H}_{2} \mathrm{O}_{2}\right)$, and potassium carbonate $\left(\mathrm{K}_{2} \mathrm{CO}_{3}\right.$, >99\%) were purchased as first grade from Wako Japan. $\mathrm{K}_{2} \mathrm{CO}_{3}$ was dried at $120{ }^{\circ} \mathrm{C}$ in vacuum overnight and stored in a desiccator before use. Anhydrous solvents were used unless stated.

SPES copolymers (Figure 1) were synthesized according to the literature.[24,37] The crude polymer solutions were precipitated in IPA, dried, protonated with $\mathrm{HCl}$, and dialyzed for $3 \mathrm{~d}$ (molecular weight cut-off (MWCO): $14 \mathrm{kDa}$, Viskase, IL, USA) to narrow their polydispersity. The dialyzed polymer solution was rotary evaporated, vacuum dried on a PTFE plate at $60{ }^{\circ} \mathrm{C}$ overnight, and the resulting polymer films were broken down with a spatula (or ground with mortars) before redrying at $60{ }^{\circ} \mathrm{C}$ overnight under vacuum. Accelerated degradation tests were carried out by adding 1.5 wt $\% \mathrm{H}_{2} \mathrm{O}_{2}$ to the polymer solutions $(2 \mathrm{mg} / \mathrm{mL})$ at $60{ }^{\circ} \mathrm{C}$. NMR, FTIR, gel permeation 
chromatography (GPC) and ion photometric chromatography were used to characterize the copolymers and the degradation by-products, as we reported previously.[24]

Unless specified, all calculations were performed at the semiempirical level of theory using the CAChe WorkSystems Pro 6.1.12 program (Fujitsu, Japan). Geometries were preoptimized using molecular mechanics (MM3) calculations and optimized at the PM3 level of theory, using the PRECISE keyword, to increase the convergence criteria by a factor of 100. The unrestricted Hartree-Fock method was used to calculate $\mathrm{OH}$ radical adduct stabilization energy and IRC trajectories. Transition states (TS) for IRC calculations were searched using the TRANS keyword. The TS and stable molecular conformations were characterized by the presence or absence of a single imaginary frequency. In all IRC calculations where one $\cdot \mathrm{OH}$ attacks a specific site at the SPES monomer, the TS were confirmed to connect the reactant and product molecules. The enthalpy of reaction $(\Delta H)$ was calculated as the difference between the heat of formation of the reaction product and the reactant molecules. Frontier MO levels and locations of SPES monomers, dimers, and tetramers were calculated from structures optimized at the PM5 level of theory. DFT calculations (B88-LYP/DZVP) were also performed on SPES monomers to verify the trends observed from semiempirical calculations (MO levels and locations).

\section{RESULTS AND DISCUSSION}

The structures of the SPES copolymers synthesized in this study are shown in Figure 1. We prepared six SPES copolymers having different dihydroxy comonomers: BP 
(SPES-a, number average molecular weight $\left(M_{\mathrm{n}}\right)$ : $24.7 \mathrm{k}$ ), BPS (SPES-b, $M_{\mathrm{n}}: 11 \mathrm{k}$ ), BPn (SPES-c, $\left.M_{\mathrm{n}}: 9.1 \mathrm{k}\right)$, BPA (SPES-d, $M_{\mathrm{n}}: 20$ k), TDP (SPES-e, $\left.M_{\mathrm{n}}: 28.5 \mathrm{k}\right)$, and BP6F (SPES-f, $M_{\mathrm{n}}: 29.8 \mathrm{k}$ ). All polymers were dialyzed to narrow their polydispersity index (PDI) to 1.6-1.8. The difference in the molecular weights of the samples was attributed to the electrophilic nature of the functional group (substituent) in the comonomer (e.g.: sulfone, carbonyl) influencing the polymerization rate.

The number average molecular weight $\left(M_{\mathrm{n}}\right)$ change over degradation time of all SPES copolymers $\left(1.5\right.$ wt $\left.\% \mathrm{H}_{2} \mathrm{O}_{2}, 60{ }^{\circ} \mathrm{C}\right)$ is shown on Figure 2. First, we examined SPES-ad, performed electronic structure calculations, and then examined SPES-e and SPES-f to verify the trend observed from our calculations. For the first set of copolymers (SPES-ad), SPES-b showed the highest chemical stability, followed by SPES-d, SPES-a, and SPES-c (Figure 2a). Interchain cross-linking was observed in the case of SPES-c at $t=$ $60 \mathrm{~min}$, and its degradation could not be followed at $t=120 \mathrm{~min}$ because of structural instability. PDI change of the copolymer samples over degradation time (Figure $\mathbf{2 b}$ ) suggested unzip scission as the dominant degradation pathway for SPES-b and SPES-d, with midpoint scission being the dominant degradation pathway for SPES-a and SPES-c. Previously, we showed that the GPC spectra of a copolymer undergoing unzip scission shifts to the low-molecular-weight region while retaining its unimodal shape, and GPC spectra of a copolymer undergoing midpoint scission shifts abruptly to lower molecular weight region, significantly broadens, and becomes multimodal.[24] Interestingly, although SPES-e showed the weakest chemical durability (Figure 2a), its degradation profile was more similar to copolymer samples undergoing unzip scission. We also noted that despite having fluorinated groups, the chemical durability of SPES-f was lower than 
that of SPES-d.

The promise of tuning the chemical durability of SPES copolymers by tailoring their molecular structures motivated us to investigate factors determining the dominant chain scission modes. We employed a semiempirical level of theory (PM3, unrestricted Hartree-Fock) to calculate the stabilization energy of $\mathrm{OH}$ adducts at several different sites at the SPES-a repeating unit (no substituent, Figure 3a). Roduner and Schlick showed that $\bullet \mathrm{OH}$ radicals are extremely reactive and attack many places, such as site 1 , (sulfonic acid group) and 5 or 6 (ether bridge), to give sulfonic acid, phenoxyl, and phenyl radicals.[34] They further showed that sulfonic acid group dissociation because of - $\mathrm{OH}$ attacks does not proceed to a significant extent, in agreement with the literature.[24,38] Therefore, we think that susceptible sites at the dihydoxycomonomers are the primary cause of midpoint scissions and the subsequent cascade reactions.[20] Figure 3a shows that $\bullet \mathrm{OH}$ was relatively stable by adding to ether bonds (sites 5 and 6 ), and to aromatic rings (sites 2 and 8) when compared with other sites. Therefore, the degradation pathway is more likely to proceed through $\bullet \mathrm{OH}$ attacks on ether bridges and aromatic rings than on sulfonic acid and sulfonic bonds.

Next, IRC calculations were carried out to compare three types of $\bullet \mathrm{OH}$ attacks: $-\mathrm{SO}_{3} \mathrm{H}$ dissociation, $-\mathrm{SO}_{2}-$ cleavage, and ether cleavage (Figure 3b). IRC calculations did not show any significant difference in the energy barrier to reach the transition state between these $\bullet \mathrm{OH}$ attacks, confirming the high reactivity of $\bullet \mathrm{OH}$. However, an appreciable difference was seen in the enthalpy of reaction of the ether cleavage compared with sulfonic acid, and sulfonic bond cleavages. Our result suggests that, indeed, bond cleavages of SPES copolymers due to ROS attacks are more likely to occur on their ether 10 
bonds.[20]

We hypothesized that the susceptibility of the copolymers to $\bullet \mathrm{OH}$ attacks can be estimated from their HOMO level because of the electrophilic nature of $\bullet \mathrm{OH} .[39,40]$ In such cases, the SPES copolymer having the lowest HOMO level would have the highest chemical stability. Such a hypothesis was supported by a semiempirical calculation (PM3) on a PFSA (Nafion®) model, showing a significantly lower HOMO level (-12.24 $\mathrm{eV}$ ), and the higher chemical stability of PFSA-PEM relative to HC-PEM is well documented.

Semiempirical theory (PM3) calculations on SPES monomers (including dimers and tetramers to eliminate the terminal atom effect) showed HOMO levels in the range of 10.02 to $-8.9 \mathrm{eV}$ (Figure 4a) We observed a moderate correlation between HOMO energy level and the $\mathrm{M}_{\mathrm{n}}$ of the copolymers at $t=60 \mathrm{~min}$ in accelerated degradation tests $\left(M_{\mathrm{n} 60 \mathrm{~min}}\right.$, Figure 4). A similar trend was observed when we used DFT (B88-LYP/DZVP) to calculate the HOMO level of the same SPES copolymers (Figure $\mathbf{4 b}$ ). The difference in the MO levels between semiempirical and DFT calculations is well documented.[41] We noted the precision issues in using semiempirical theory and the less-strict basis set of DFT to compute the MO level of oligomers; however, the similar trend from both calculation methods provides sufficient support for the chemical durability difference within SPES copolymers.

Indeed, SPES-b has a higher oxidative stability and lower HOMO level $(-10.02 \mathrm{eV})$ than other SPES copolymers (e.g., the HOMO of SPES-a is $-9.36 \mathrm{eV})$. However, SPESc has lower oxidative stability than SPES-a despite having a lower HOMO level ( -9.59 
eV) (Figure 4). Such low oxidative stability of SPES-c was attributed to the radical susceptibility of the benzophenone moiety of the dihydroxy comonomer.

Further, we found that SPES-d (HOMO: $-9.25 \mathrm{eV}$ ) has higher chemical stability than SPES-a (HOMO: $-9.36 \mathrm{eV}$ ) despite its higher HOMO level. Because the structural difference between SPES-a and SPES-d is the propanediyl group of the dihydroxy comonomer of SPES-d, this result suggested the role of steric groups in hindering ROS attacks on the polymer backbone or preventing cascade reactions after ROS attacks on the aromatic rings. We then examined the significance of HOMO level and steric groups in increasing the chemical stability of the SPES copolymers.

We synthesized SPES-e and SPES-f and evaluated their oxidative stability together with SPES-a-d (Figure 2). SPES-e was reported to be a promising HC-PEM material because of its high proton conductivity.[42] SPES-f was expected to have higher ROS resistance than SPES-d because of its partially fluorinated substituents. The HOMO level of SPES-e $(-8.95 \mathrm{eV})$ and the comonomer structure (no steric groups) suggested its chemical stability to be lower than SPES-a, b, $\mathbf{c}$, and d. Indeed, SPES-e exhibited the weakest chemical durability among other SPES copolymers (Figure 2a, 4). In the case of SPES-f, the fluorinated substituent in the comonomer is attributed to lowering the HOMO level of the polymer $(-9.88 \mathrm{eV})$ and adding steric hindrance to the polymer backbone. Therefore, its chemical stability was expected to be higher than SPES-a, c, d, and $\mathbf{e}$, and comparable or slightly lower than SPES-b. The oxidative stability of SPES-f was found to be higher than SPES-a, c, and e, and lower than SPES-b. However, the oxidative stability of SPES-f was lower than that of SPES-d and much lower than SPESb (given the difference in their HOMO levels). Although HOMO levels and steric groups 12 
proved useful to estimate the chemical stability of SPES, these two factors could not explain the midpoint scission process and low chemical stability of SPES-c (despite its low HOMO level) and the relatively low chemical stability of SPES-f (despite having fluorinated steric groups).

We generated and optimized the structure of all SPES copolymers as dimers and tetramers, calculated their frontier MOs, and visualized their locations. Figure 5 shows that the HOMOs of SPES-a, b, d, and e are located at their dihydroxy comonomers, their LUMOs are located at the sulfonic acid or arylsulfonic acid groups, and both MOs are located near the chain ends. Such a trend may account for the unzip scission mechanism as the dominant chain scission mode for these SPES copolymers. We also noted that the HOMO location at the aromatic rings and ether bridges agreed with calculation results described in the previous sections. Interestingly, in the case of SPES-e, the HOMO density is higher on the sulfur group than on the aromatic carbons of the TDP comonomer. This result suggests a higher rate of unzip scissions because of the preference of $\bullet \mathrm{OH}$ to attack the thioether group rather than the aromatic rings, and is presumably, at least in part, the rationale for the low oxidative stability of SPES-e. Such a unique difference between SPES copolymers cannot be deduced from experiments and their HOMO level calculations.

Furthermore, the locations of HOMOs and LUMOs for SPES-c and SPES-f were located at their dihydroxy comonomers with slight overlapping at the substituents (e.g., the benzophenone component of SPES-c) and the aromatic rings (Figure 5, marked with asterisk symbols). The LUMOs of SPES-c and SPES-e for both dimers and tetramers were located in the middle of the polymer chains. Such a trend may account for midpoint 13 
scission as the dominant chain scission mode for these SPES copolymers. The relatively low chemical stability of these copolymers (despite their low HOMO levels) is presumably attributed to additional simultaneous attacks from other reactive species (HOO•, nucleophiles). The benzophenone moiety of SPES-c is susceptible to nucleophilic attacks and substitutions. Bisphenol 6F moiety of SPES-f can be attacked by $\mathrm{H}_{2} \mathrm{O}_{2}$ or $\mathrm{HOO} \cdot$ to give benzene and an arylhexafluoropropyl radical intermediate. This intermediate can react with $\mathrm{H}_{2} \mathrm{O}_{2}$ to give an aryl radical and hexafluoroacetone, a highly reactive electrophile that can trigger cascade reactions.[43]

\section{CONCLUSIONS}

In summary, accelerated degradation tests and electronic structure calculations were performed to examine factors influencing the chemical durability of SPES copolymers in a highly oxidative environment. The dihydroxy comonomer structure of SPES copolymers influences the dominant chain scission modes of the SPES copolymers. Lowering the HOMO levels of the copolymer improves its chemical stability because of increased energy barrier for ROS to abstract electrons from the polymer. However, consideration of steric effects and MO locations of the copolymers can improve the prediction of their chemical stability. The presence of steric groups can hinder ROS attacks on the polymer backbone, and MO location analysis allow for insights into the dominant chain scission modes of the polymers. Reducing the electron density of the polymer backbone, attaching bulky steric groups, and diverting main chain attack (e.g., attaching sacrificial radical-trapping functional groups) are expected to increase the oxidative stability of SPES and similar sulfonated hydrocarbon-based polymers. Rational design of copolymers to suppress chemical degradation by correlating the ROS resistance 14 
of polymeric materials to their electronic structures may prove useful as a new platform to design highly robust PEMs for fuel cells and other high-temperature applications.

\section{Acknowledgments}

The authors thank the New Energy and Industrial Technology Development Organization, Japan, and the Kanagawa Academy of Science and Technology, Japan, for their support for this research.

\section{AUTHOR INFORMATION}

\section{Corresponding Author}

*E-mail: yamag@res.titech.ac.jp (T.Y.)

\section{REFERENCES}

[1] Wang, Y.; Chen, K. S.; Mishler, J.; Cho, S. C.; Adroher, X. C. Appl. Energy 2011, 88, 981-1007.

[2] US Department of Energy. Fuel Cell Technologies Office: Multi-Year Research, Development, and Demonstration Plan. http://energy.gov/eere/fuelcells/fuel-celltechnologies-office-multi-year-research-development-and-demonstration-plan (accessed Jan 21, 2014) 
[3] Chandan, A.; Hattenberger, M.; El-kharouf, A.; Du, S.; Dhir, A.; Self, V.; Pollet, B. G.; Ingram, A.; Bujalski, W. J. Power Sources 2013, 231, 264-278.

[4] Liu, H.; Gasteiger, H. A.; Laconti, A.; Zhang, J. ECS Transactions 2006, 1, $283-$ 293.

[5] Scherer, G. G. Ber. Bunsen-Ges. Phys. Chem. 2010, 94, 1008-1014.

[6] Pozio, A.; Silva, R. F.; De Francesco, M.; Giorgi, L. Electrochim. Acta 2003, 48, $1543-1549$.

[7] Yu, J.; Yi, B.; Xing, D.; Liu, F.; Shao, Z.; Fu, Y.; Zhang, H. Phys. Chem. Chem. Phys. 2002, 5, 611-615.

[8] Mattsson, B.; Ericson, H.; Torell, L. M.; Sundholm, F. Electrochim. Acta 2000, 45, 1405-1408.

[9] Wu, J.; Yuan, X. Z.; Martin, J. J.; Wang, H.; Zhang, J.; Shen, J.; Wu, S.; Merida, W. J. Power Sources 2008, 184, 104-119.

[10] Endoh, E.; Terazono, S.; Widjaja, H.; Takimoto, Y. Electrochem. Solid-State Lett. 2004, 7, A209.

[11] Paddison, S. J. Annu. Rev. Mater. Res. 2003, 33, 289-319.

[12] Borup, R.; Meyers, J.; Pivovar, B.; Kim, Y. S.; Mukundan, R.; Garland, N.; Myers, D.; Wilson, M.; Garzon, F.; Wood, D.; Zelenay, P.; More, K.; Stroh, K.; Zawodzinski, T.; Boncella, J.; McGrath, J. E.; Inaba, M.; Miyatake, K.; Hori, M.; Ota, K.; Ogumi, Z.; Miyata, S.; Nishikata, A.; Siroma, Z.; Uchimoto, Y.; Yasuda, K.; Kimijima, K.-I.; Iwashita, N. Chem. Rev. 2007, 107, 3904-3951.

[13] Lee, J.; Marvel, C. S. J. Polym. Sci. Polym. Chem. Ed. 1984, 22, 295-301.

[14] Kim, Y. S.; Einsla, B.; Sankir, M.; Harrison, W.; Pivovar, B. S. Polymer 2006, 
47, 4026-4035.

[15] Okada, T. J. Electroanal. Chem. 1999, 465, 1-17.

[16] Kerres, J.; Ullrich, A.; Hein, M.; Gogel, V.; Friedrich, K. A.; Joerissen, L. Fuel Cells 2004, 4, 105-112.

[17] Hickner, M.; Ghassemi, H.; Kim, Y.; Einsla, B.; McGrath, J. Chem. Rev. 2004, 104, 4587-4611.

[18] D. S. Kim, G. P. Robertson, Y. S. Kim, M. D. Guiver, Macromolecules 2009, 42, 957-963.

[19] Zhang, H.; Shen, P. K. Chem. Rev. 2012, 112, 2780-2832.

[20] Collier, A.; Wang, H.; Yuan, X. Z.; Zhang, J.; Wilkinson, D. P. Int. J. Hydrogen Energy 2006, 31, 1838-1854.

[21] Park, C. H.; Lee, C. H.; Guiver, M. D.; Lee, Y. M. Prog. Polym. Sci. 2011, 36, $1443-1498$.

[22] Prabhakaran, V.; Arges, C. G.; Ramani, V. Proc. Natl. Acad. Sci. USA 2012, 109, $1029-1034$.

[23] Trogadas, P.; Parrondo, J.; Ramani, V. Electrochem. Solid-State Lett. 2008, 11, B113-B116.

[24] Lawrence, J.; Yamaguchi, T. J. Membr. Sci. 2008, 325, 633-640.

[25] Harrison, W. L.; Wang, F.; Mecham, J. B.; Bhanu, V. A.; Hill, M.; Kim, Y. S.; McGrath, J. E. J. Polym. Sci., Part A: Polym. Chem. 2003, 41, 2264-2276.

[26] Schuster, M.; Kreuer, K.-D.; Andersen, H. T.; Maier, J. Macromolecules 2007, 40, 598-607.

[27] Miyatake, K.; Furuya, H.; Tanaka, M.; Watanabe, M. J Power Sources 2012, 
204, 74-78.

[28] Miyatake, K.; Zhou, H.; Uchida, H.; Watanabe, M. Chem. Commun. 2003, 368369.

[29] Miyatake, K.; Chikashige, Y.; Watanabe, M. Macromolecules 2003, 36, 96919693.

[30] Aoki, M.; Chikashige, Y.; Miyatake, K.; Uchida, H.; Watanabe, M. Electrochem Commun 2006 8, 1412-1416.

[31] Y. S. Kim, L. Dong, M. A. Hickner, T. E. Glass, V. Webb, J. E. McGrath, Macromolecules 2003, 36, 6281-6285.

[32] Stewart, J. J. P. J. Mol. Model. 2004, 10, 155-164.

[33] Friesner, R. A.; Guallar, V. Annu. Rev. Phys. Chem. 2005, 56, 389-427.

[34] Fukui, K.; Yonezawa, T.; Shingu, H. J. Chem. Phys. 1952, 20, 722-725.

[35] Gutteridge, J. M. Biochem. J. 1987, 243, 709-714.

[36] Cheng, Z.; Ren, J.; Li, Y.; Chang, W.; Chen, Z. Bioorg. Med. Chem. 2002, 10, 4067-4073.

[37] Parra, S.; Olivero, J.; Pulgarin, C. Appl. Catal., B 2002, 36, 75-85.

[38] Roduner, E.; Schlick, S. In Advanced ESR Methods in Polymer Research; John Wiley \& Sons, Inc., 2006; pp. 197-228.

[39] Raghavan, N. V.; Steenken, S. J. Am. Chem. Soc. 1980, 102, 3495-3499.

[40] Marusawa, H.; Ichikawa, K.; Narita, N.; Murakami, H.; Ito, K.; Tezuka, T. Bioorg. Med. Chem. 2002, 10, 2283-2290.

[41] Yang, S.; Olishevski, P.; Kertesz, M. Synth. Met. 2004, 141, 171-177.

[42] Gross, M.; Maier, G.; Fuller, T.; MacKinnon, S.; Gittleman, C. Handbook of 
Fuel Cells; John Wiley \& Sons, Inc., 2010; pp 1-17.

[43] Howard, E. G.; Sargeant, P. B.; Krespan, C. G. J. Am. Chem. Soc. 1967, 89, $1422-1430$. 


\section{FIGURES}

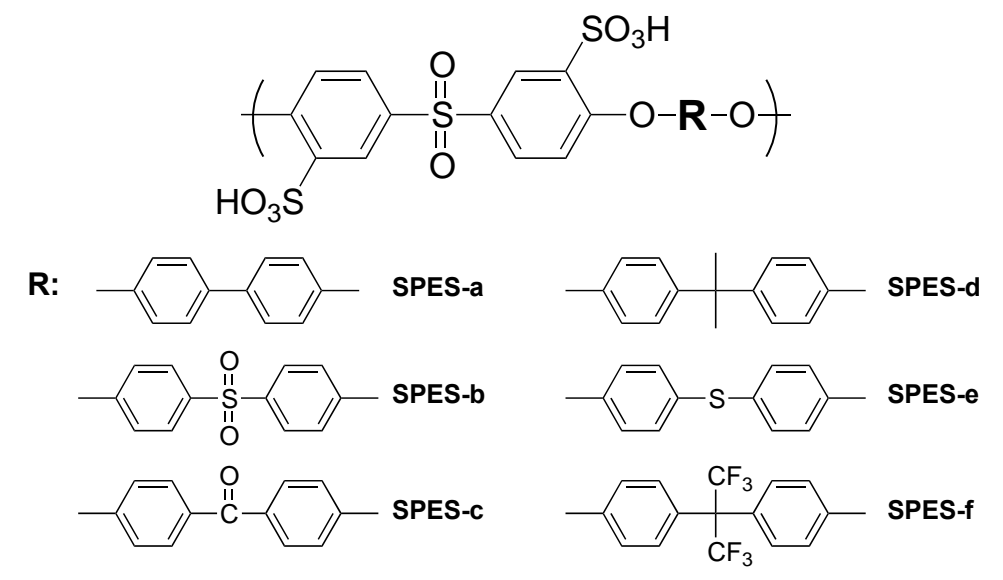

Figure 1. SPES copolymers evaluated in this study. R: comonomer structures. 

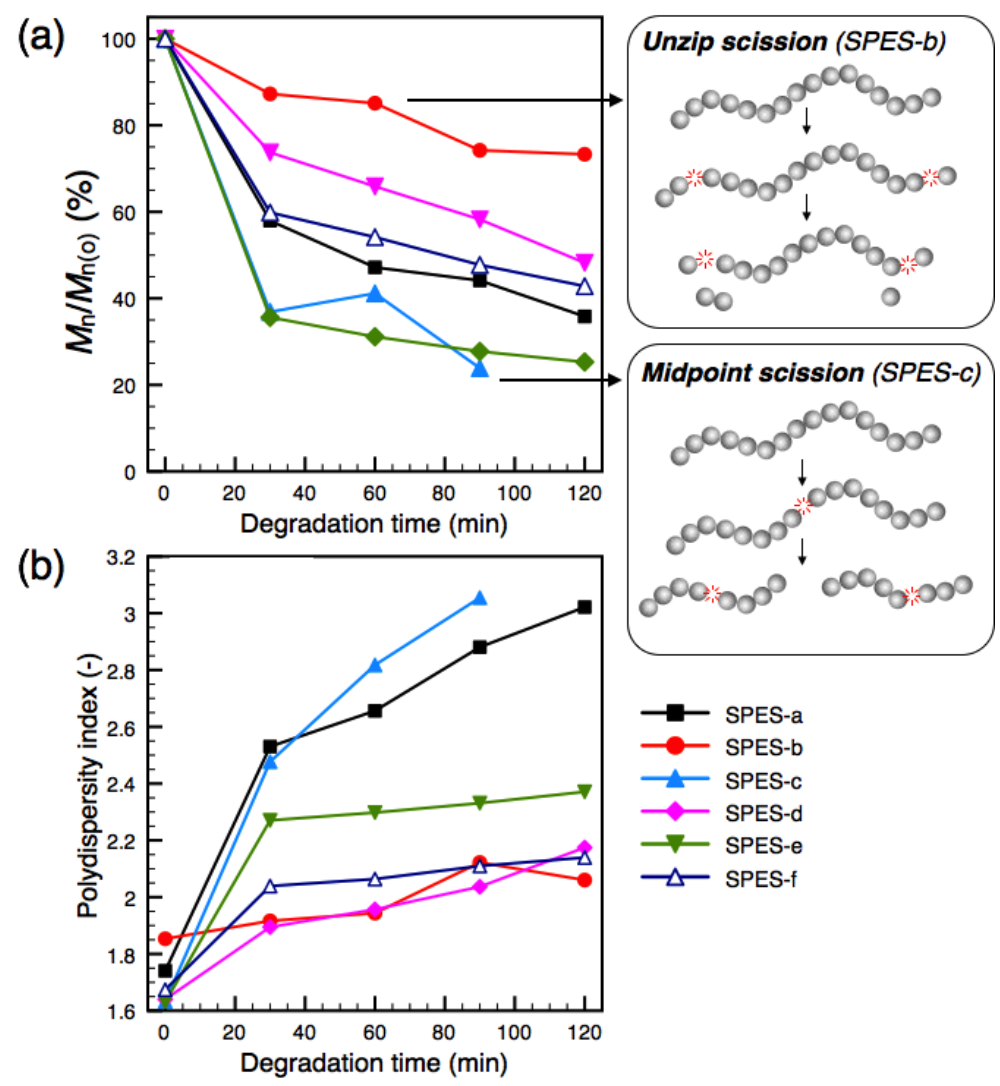

Figure 2. (a) Normalized $M_{\mathrm{n}}$ and (b) $M_{\mathrm{w}} / M_{\mathrm{n}}$ (PDI) of SPES a-f plotted over degradation time. 
(a)

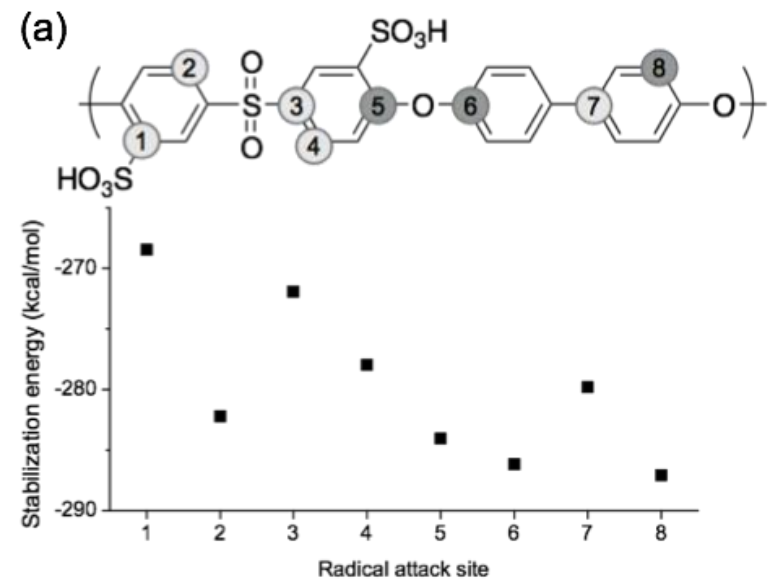

(b)

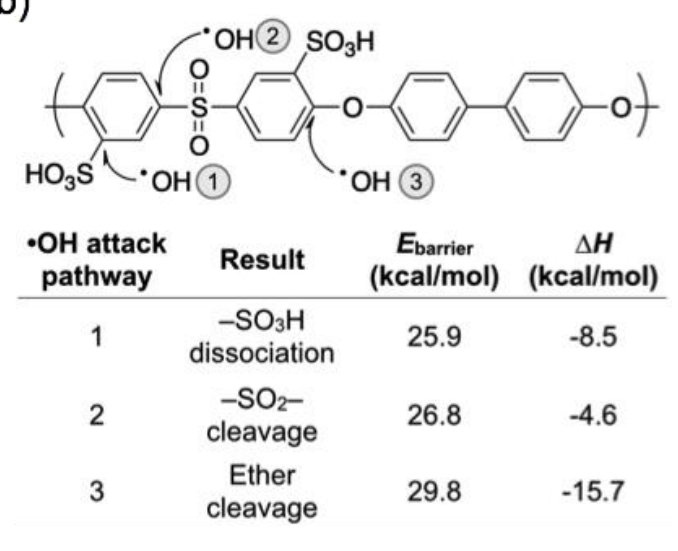

Figure 3. (a) Stabilization energy of $\mathrm{OH}$ radical adducts of SPES-a, calculated using semiempirical theory (PM3). Sites having relatively low stabilization energy for $\mathrm{OH}$ radical adduct are marked with dark circles. (b) IRC calculation of $\mathrm{OH}$ radical attacks on sulfonic acid bond, sulfonic bond, and ether bond of SPES-a. 

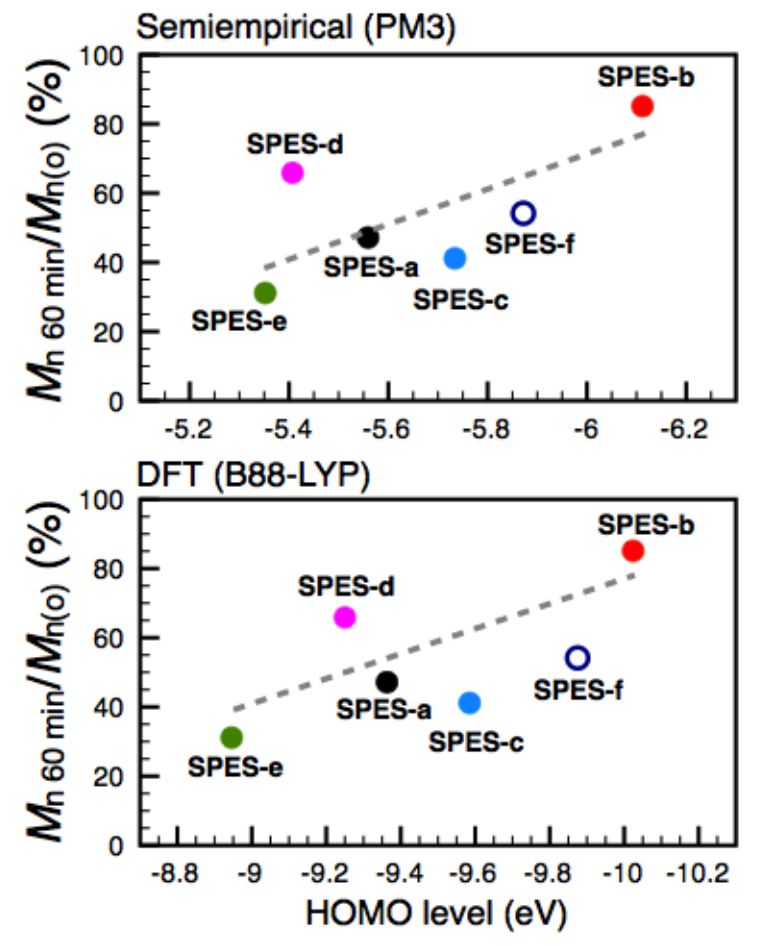

Figure 4.The normalized $M_{\mathrm{n}}$ after 60 min of accelerated degradation test $\left(M_{\mathrm{n}} 60 \mathrm{~min}\right)$ and the HOMO of SPES-a-f calculated at the (a) PM3 and (b) B88-LYP/DZVP level of theory. The correlation between the two variables is shown with a dashed gray line. 


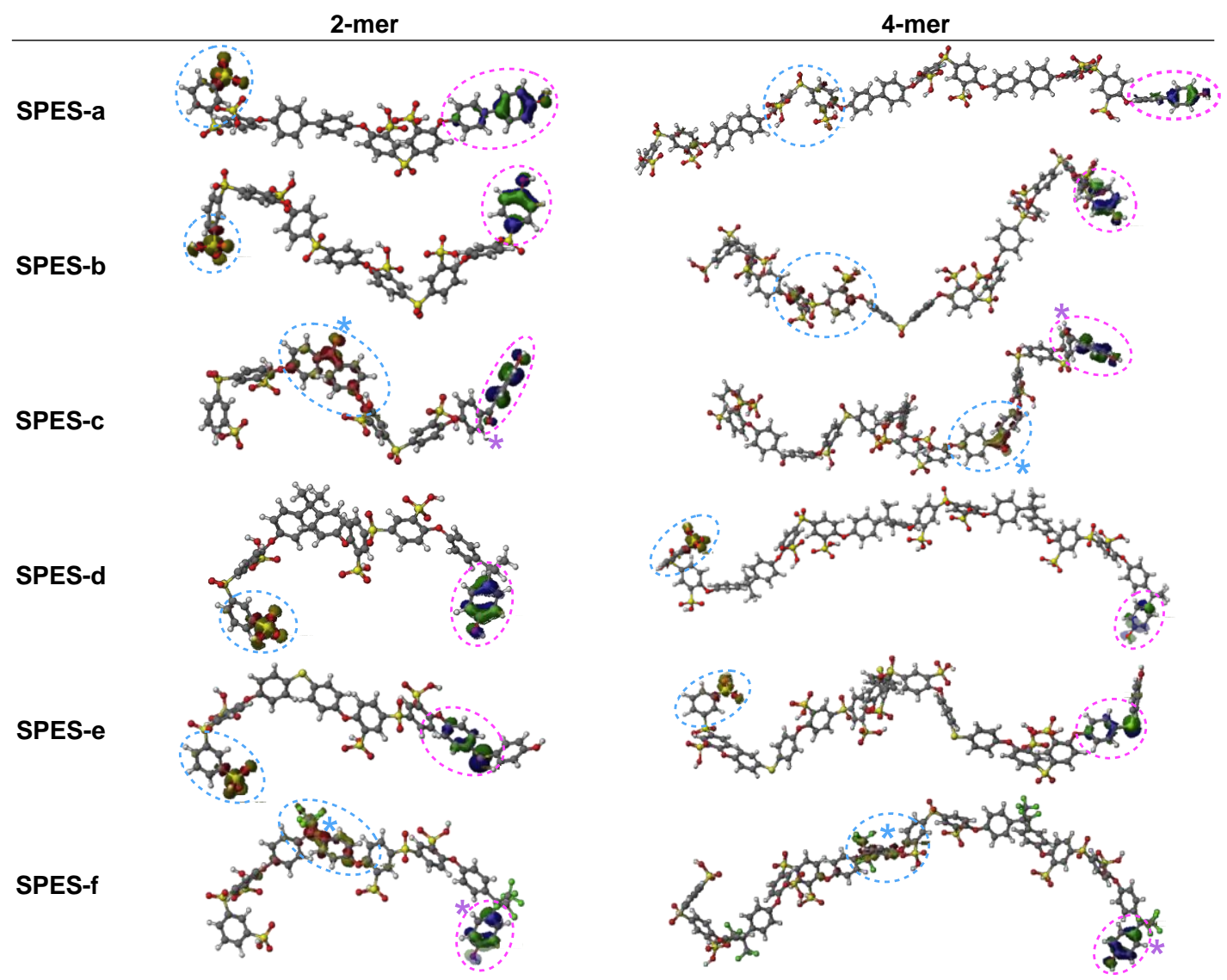

Figure 5. The molecular structures of SPES-a-f oligomers (dimers and tetramers) in their most stable conformations. For all structures, the HOMO is shown in blue-green and encircled with a magenta dashed circle for visual aid. The LUMO is shown in red-yellow and encircled with a cyan dashed circle for visual aid. Dihydroxy comonomer sites where HOMO and LUMO overlap are marked with cyan and magenta asterisks (SPES-c and SPES-f). 


\section{Graphical Abstract}

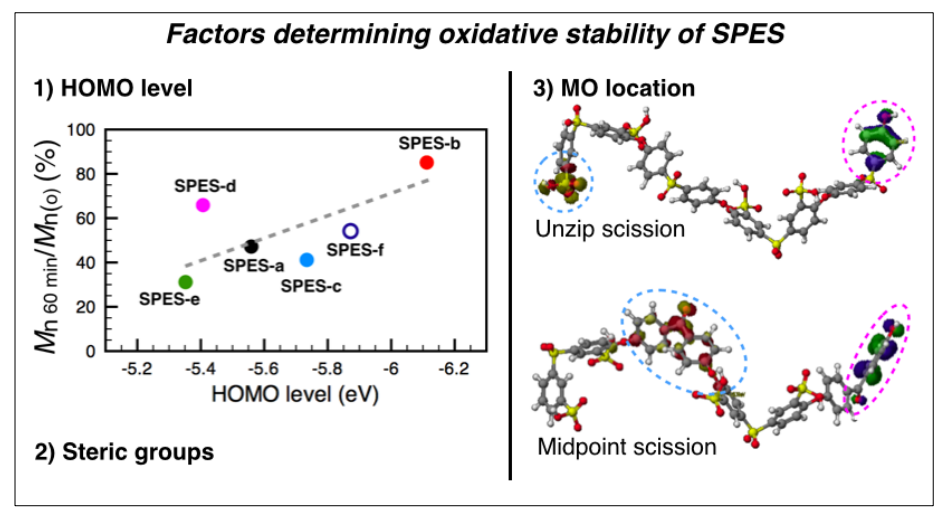

\section{Correlating Electronic Structure and Chemical Durability of}

\section{Sulfonated Poly(Arylene Ether Sulfone)s}

Jimmy Lawrence, Koichi Yamashita, Takeo Yamaguchi 